\title{
The Boyd Conjecture
}

\author{
Jeffrey H. Boyd \\ Retired, 57 Woods Road, Bethany, CT 06524, USA \\ JeffreyHBoyd@gmail.com
}

\begin{abstract}
The Boyd Conjecture is that the amplitude of an elementary ray from the Theory of Elementary Waves (TEW) is the physical analog of a probability amplitude from Quantum Mechanics (QM). Boyd learned TEW from his cousin Lewis E. Little, who had said that quantum math is the roadmap to the world of elementary waves, but we didn't know how to read the map. He suggested that Boyd, with a degree in mathematics, should try to decipher the hieroglyphic map. In 2014 Boyd began thinking that amplitudes are the core of QM, and elementary rays the core of TEW: perhaps they were equivalent. Both decisively influence particle behavior without conveying any energy. There are no other examples of zero energy waves having a huge impact on a particle's behavior. This essay discusses the history, strengths, weakness and implications of this conjecture. This is the first time that quantum math is not abstract, but is grounded on the physical reality of the classical world of 3 dimensions in which we live.
\end{abstract}

\section{Indexing terms/Keywords}

Foundations of quantum mechanics, probability amplitudes, Theory of Elementary Waves.

\section{Academic Discipline And Sub-Disciplines}

Physics, quantum physics, quantum mathematics;

\section{SUBJECT CLASSIFICATION}

Library of Congress Classification \#'s for Quantum Theory are from QC173.96 to QC174.52

for example: QC173.96 Quantum Mechanics Foundations

$$
\begin{aligned}
& \text { or QC174.12.Q36 Quantum theory } \\
& \text { or QC174.2NT Wave Particle Duality }
\end{aligned}
$$

\section{TYPE (METHOD/APPROACH)}

This article records the history of how the Boyd Conjecture came into existence, along with the meaning of that Conjecture in the context of TEW. We seek to provide a QM explanation of elementary rays and a TEW explanation of quantum amplitudes.

\section{INTRODUCTION}

A conjecture is a statement which appears to be true, but has not been formally proved. The term comes from the vocabulary of mathematicians. An elementary ray is the centerpiece of the Theory of Elementary Rays, a radical new idea in which particles follow elementary rays backwards.

Much of math and science is constructed in the dark, or in a fog, without clear sight of how nature is organized. The community of scientists have ways of thinking that are often helpful; but when it comes to a paradigm shift, those thought habits can be worse than knowing nothing at all. This article pertains to a major paradigm shift in physics. Consider the fate of a paradigm shift of similar magnitude in the past.

Geology was considered to be a robust and open minded science in the early twentieth century. In 1912 Alfred Wegener proposed that all the continents had once been joined in a super-continent for which he coined the term "Pangea," that split up, and continents had been drifting apart since. His proposal was universally condemned as nonsense by all the leading geologists and scholarly societies, was not taught in any textbooks, and dropped out of human history until long after Wegener died twenty years later. Even his father in law said his idea was stupid. Wegener's "stupid idea" re-emerged at the core of a new paradigm: tectonic plate theory. Thomas Kuhn says that a paradigm shift involves such a divergent way of thinking that the leading scientists who are invested in the previous paradigm, dismiss the new idea as unintelligible gibberish.(1)

Quantum mechanics is a well developed and universally accepted theory of subatomic world, which has been more accurate and productive than any other science has ever been. QM can be thought of as a body of mathematics connected to a way of thinking about how nature is organized. It is the math that has done the heavy lifting. The QM picture, or non-picture of nature is weird, as everyone knows: fraught with contradictions and brain-twisters. Everyone can agree there is something wrong; no one can agree how to fix it.

\section{History of the Boyd Conjecture}

Lewis E. Little was a brilliant student who graduated magna cum laude from Brown University in 1962 majoring in applied math and physics. This author is Little's cousin, and followed Little into the applied math 
department of Brown based on Little's enthusiasm. Little was always an argumentative guy, and was strongly influenced by the writings of Ayn Rand, an argumentative author. Boyd dislikes Ayn Rand, and is not fond of arguments. Upon graduating from college Little was puzzled by quantum mechanics, and told Boyd he wanted to figure out whether QM was crazy or he was crazy.

To answer that question Little went to graduate school in the physics department at Princeton University. After a few years he concluded that QM was crazy (meaning the metaphysics or worldview of QM). He was given a masters degree, but then got his PhD in physics from New York University in 1974. By that time Little had embarked on a long career trading commodities on the stock market. He wanted to teach physics, but his disagreement with QM made that career impossible.

To say there is something weird about QM is commonplace, even among quantum physicists. Little set out to build a better theory, one that could explain the experimental data without the weirdness. For three decades he read and pondered physics. He was isolated, alone, working in his spare time on weekends and nights: no one to talk to. He filled notebooks with his ideas and found that every five years he cycled through the same speculations and ruminations.

Finally in 1992 he had an idea that had never occurred to anyone in human history: that particles follow waves backwards. It was a peculiar idea. This idea explained a myriad of quantum experimental phenomena. It eliminated quantum weirdness. He said it also explained relativity. When he wrote a scholarly article, most physics journals rejected it, saying, in essence, that his idea was as stupid as Wegener's.(2)

Boyd first heard about TEW at at his aunt's $80^{\text {th }}$ birthday party in 2000 . Stephen Speicher, a physicist who was also a follower of Ayn Rand, was the primary proponent of TEW and arranged for Little to speak at the Jet Propulsion Labs at CalTech in 2000 (talks available on YouTube).(3) Tragically, Speicher died in 2007.

After a long career in medicine, including many presentations at scholarly conventions and publications in peer reviewed medical journals (including the leading medical journal, New England Journal of Medicine) and years on the faculty of the National Institutes of Health in Bethesda, Boyd joined the American Physical Society in 2010 and began dragging Little to APS meetings. The purpose was for Little to answer questions physicists might have after Boyd's presentation on TEW. In those days Boyd had the naive idea that if you can to talk to physicists in their own language about an idea that improved ones thinking about quantum weirdness, then the physicists would be swayed. Little published a book in 2009 the first paragraph of which more-or-less says that all academic physicists are fools.(4)

Boyd presented TEW to APS conventions up to four times every year for seven years. He began publishing scholarly articles in Physics Essays in 2012.(5,6) In 2016 his wife told him he should stop wasting time presenting at APS meetings, since it was like talking to a brick wall.

Boyd has a way of stating the blunt truth and letting the chips fall where they may, a different style than Little who prefers to think through every angle and sometimes takes years before he feels that an idea is ready to be published. That difference came to a crisis in 2013 when Boyd proceeded to publish a TEW explanation of the Bell test experiments (giving Little all the credit) before Little wanted it published. Speicher had told Boyd that only if the Bell test experiments could be explained, would TEW gain credibility. A severe argument erupted in which Little promised to go to a lawyer and sue Boyd, unless the article was withdrawn before publication. Boyd did not back down.(7)

What was this dispute about? In Boyd's view it was about ownership of TEW. Little spoke of TEW as belonging to himself. He wanted to make sure every detail was right and invulnerable to attack. Boyd recalls Little speaking of growing wealthy from proprietary rights. In Boyd's view TEW belonged to the scientific community and/or the human race, and our responsibility was to disseminate those ideas as quickly as possible, even if we made some blunders. Boyd viewed science as a community effort, not the effort of any one isolated genius. Boyd was far more willing to make a mistake and look like a fool in public than was Little. The two men have not spoken since December 2013.

After that rupture with Little, Boyd continued to feel that TEW was too important to allow it to die out with our generation. For a while it looked as if TEW would vanish from the face of the earth when we die of old age. In 2017 Little is 75 and Boyd 73 years old. Boyd continued to speak, publish, and make YouTube videos.(8-15)

Boyd continued to puzzle about how TEW relates to quantum math. For years he wrestled with questions such as how the Dirac equation or Klein-Gordon equation related to TEW. He explored how the Dirac gamma matrices could relate to TEW, and discovered nothing. That line of inquiry led to a dead end.

\section{Strengths of the Boyd Conjecture}

Math often starts with the smallest question: "What is the unit of analysis?" For example, before you ponder a numeric issue, you must postulate that there are numbers, and those numbers refer to something in the physical world. The simplest idea in TEW is the existence of elementary rays.

In QM the simplest idea is amplitudes, which are complex numbers. There is a well known mystery in QM: what do the amplitudes refer to in the physical world? You could answer that amplitudes are the square root of probabilities, but then you have evaded the question. QM is the only science that uses amplitudes: all other

4831 | P a g $\mathrm{C}$ 
sciences use probabilities. Why? What is the physical analog of an amplitude? No one had been able to answer that question. Hundreds of geniuses (including dozens of Nobel laureates) have pondered this mystery and been unable to solve it. As a result QM is taught as an abstract theory disconnected from physical reality, except for "observables" that drop out of the equations.

\section{2a. Feynman's book QED}

The possibility that an elementary ray is somehow the same thing as a quantum amplitude, intrigued Boyd for years. The first chapter of Feynman's book QED talks about "little arrows" that photons follow. These arrows spin like the hand of a one-handed clock as they move out from the lightbulb. Feynman didn't want to scare the lay public by speaking of complex numbers. So he simplified complex amplitudes by calling them "tiny arrows." We all recognize amplitudes in this formula: $A=|A| e^{\wedge} i \theta$. Feynman had simply taken $|A|$ as being the length of a "tiny arrow" and $\theta$ as the angle of rotation. He said the probability of a photon traveling in that direction is the length of the arrow squared.(16)

Feynman's path integral approach involves adding together all the amplitudes that connect a light source to a target, and thereby calculating the total amplitude that light will reach the target.

As Boyd read Feynman, he had in his head the picture of these spinning "little arrows" as shown in Figure 1. Boyd was trying to simplify and so he focused on just one of Feynman's amplitudes, ignoring the myriad of other amplitudes that Feynman emphasized.

From that picture it is only a tiny step to turning these corkscrews around so that they all impinge on the lightbulb (change direction of red arrow in Fig 1). This does not change Feynman's math one iota. Feynman had no evidence about which way the corkscrews travel. In TEW photons would follow these corkscrews backwards. By such a tiny change to Feynman's picture you would obtain a diagram of an elementary ray: moving from the detector to the particle source.

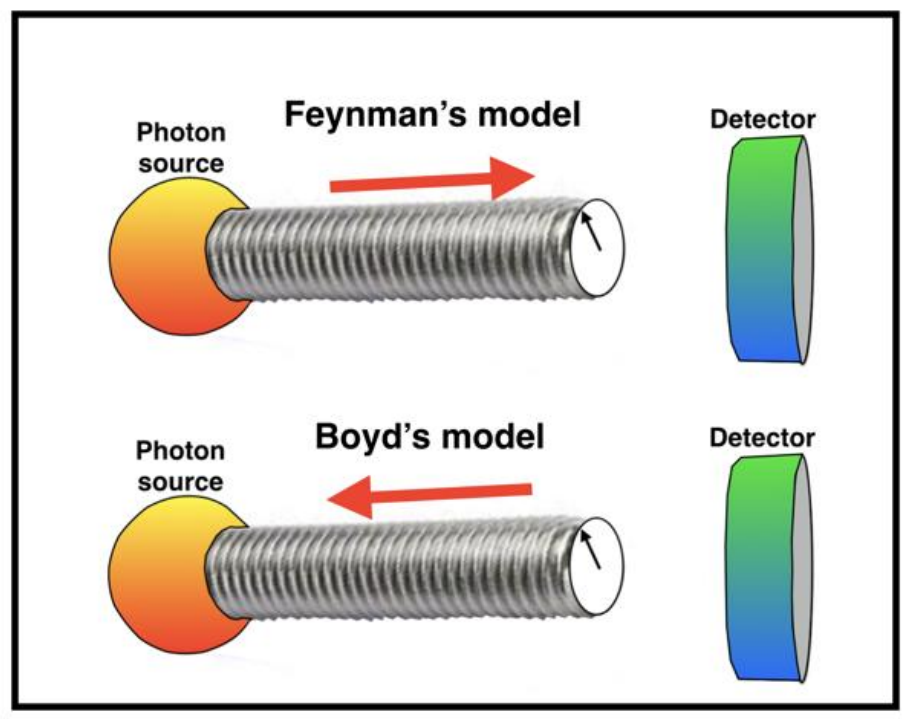

Figure 1 compares the Feynman and Boyd models of how an amplitude works in space. The amplitude consists of the tiny arrow on the clock face (at the end of the helix). For orange light the wavelength (horizontal distance from one rotation to the next) would be about $600 \mathrm{~nm}$. The arrow spins very fast. This amplitude squared is the probability that a photon will take that particular route from the source to the detector. Feynman's emphasis is that there are many such trajectories (corkscrews), some of them crooked. Feynman wants to add all the different amplitudes together, whereas Boyd selects just one of them and focuses on how it works. In the Boyd model everything is the same as in Feynman except for the direction of movement (the red arrow). Thus the math is identical, namely $A=|A| e^{\wedge} i \theta$ is the amplitude of a photon taking that trajectory.

Feynman assumes that the corkscrew starts at the source and goes toward the detector, but offers no proof, and no explanation about why he makes that assumption. Boyd names the corkscrew (both the spinning "little arrow" and its movement toward the photon source) an "elementary ray ÆE." In both models the amplitude profoundly affects the direction of the photon even though the amplitude conveys ZERO energy.

The elementary ray needed a name, so Boyd coined $Æ$. This ray ( $/$ ) is more complicated than the amplitude $\left(A=|A| e^{\wedge} i \theta\right)$ of the ray, because $Æ$ also involves the center of the one-handed clock moving at the speed of light. Thus the complex vector space does not involve $Æ$ as linear vectors, but the amplitudes of $Æ E$ as linear complex vectors. Boyd also coined $\Pi$ as the name of a particle separate from an elementary ray (however in nature there is never found a particle that is separate from all elementary rays). 
This manner of thinking leads immediately to the definition of an adjoint: $A^{*}=\left|A^{*}\right| e^{\wedge}(-i \theta)=|A| e^{\wedge}(-i \theta)$. Therefore a right handed corkscrew has a left handed corkscrew as its adjoint, i.e. its mirror image.

\section{2b. For Hilbert space you need an inner product}

For years Boyd despaired of finding the doorway between the three dimensional Cartesian space we live in and the n-dimensional Hilbert space where amplitudes live. The Hilbert space of QM led many brilliant people to say that quantum math was intrinsically abstract and that what Boyd wanted to do (find the physical foundations of QM math) could not be done.

The central issue was defining an inner product. An inner product can, with one exception, be defined once we have defined an adjoint.

\section{2c. Wave particles have " $n$ " levels of allowed energy}

The one exception is that we have to figure out how it happens that this Hilbert space has "n" dimensions, with "n" ranging as high as infinity. Cartesian space is limited to 3 dimensions. In order to solve this problem we need to take a side excursion through the wave particle partnership.

In TEW the "wave particle" of QM is split asunder and the wave travels in the opposite direction (coming from the target). The wave carries zero energy. All energy and momentum is in the particle. The reason a particle follows an elementary ray is because that is how nature is rigged up. Why does a person who wants to walk in the woods follow a path? The path contributes no energy to the hiker's walk. Hikers are more likely to follow a broad path than to bushwhack or follow a narrow path. In the metaphor, the hiker represents a particle.

Why does a photon follow one of Feynman's corkscrews? The corkscrew offers zero energy. Some corkscrews are fat and are more likely to be followed, whereas other corkscrews a slender and less likely to be followed. Whatever answer you give for why Feynman's photons follow Feynman's "little arrows," is exactly the same reason why a particle follows the corkscrews of TEW.

We cannot see a ray without a particle (which is the vast majority of rays). Because it has zero energy, a particle-less ray cannot make a detector "click." We are immersed in a vast ocean of invisible rays that probably have been around since the Big Bang. Wave-particles are the actual units of analysis, the simplest thing which we can observe in experiments. To reiterate: the simplest thing we can observe is the complex amplitude of $Æ+$ $\Pi$. We cannot observe $Æ$ without $\Pi$. The wave-particle $(Æ E+\Pi)$ moves in the direction of the particle, and is located where the particle $\Pi$ is located.

The first approximation of the Boyd Conjecture is that an elementary ray is the physical analog of a probability amplitude. The second approximation is that the amplitude (a complex number) of an elementary ray with attached particle, is the physical analog of a probability amplitude.

Depending on its energy, every particle has a deBroglie frequency $\square=E / h$, which defines the frequency of the elementary ray that $\Pi$ is able to follow. Imagine a set of all possible energies for a particular particle $\{\mathbf{E}(n)\}$. That corresponds to a set of all the allowable wave particles $\{\Pi+\not \mathbb{E}(\mathbf{n})\}$ where the $\mathbb{E}(\mathbf{n})$ refer to cylindrical helices of different wavelengths. Thus, for the energy representation we can define such a set of many corkscrews as a Dirac ket.

Now we can finally see how we humans live in the midst of an $\mathbf{n}$-dimensional Hilbert space. We have defined an amplitude for each wave-particle $\left(A=|A| e^{\wedge} i \theta\right)$. We have defined a complex vector space, an adjoint $\left(A^{*}=|A|\right.$ $\left.\mathrm{e}^{\wedge}-(i \theta)\right), \mathbf{n}$ dimensions of energy, and therefore an inner product.

$$
A \bullet B=\sum_{n}\left|A_{n}^{*}\right| \bullet\left|B_{n}\right| \cos \left(\theta_{n}-\phi_{n}\right)
$$

We can now declare victory: we have mapped out a way in which an elementary ray is the physical analog of a probability amplitude. The only difference is they travel in opposite directions: a probability amplitude would originate at Feynman's lightbulb and move outward, whereas an elementary ray would move centripedally toward the lightbulb. We have discovered the physical foundations in everyday experience of quantum amplitudes.

\section{Weaknesses of the Boyd Conjecture}

Boyd has a weakness, which is that he will spell things out, hoping that other people will correct his errors. This leadership style depends on a collaborative team to tell him when he is making an error. In meetings he often will put forward a "proposed consensus opinion" to see who buys in and who rejects the idea. He is willing to scuttle the idea if others reject it for good reasons. In committees other participants usually experience Boyd as helpful in focusing the discussion. Committees are more productive, because it is better to debate a proposition that is wrong, than to have no proposition to bring focus to an amorphous discussion.

However, this willingness to put up trial balloons depends on the presence of an audience who is attentive, thoughtful and willing to debate. In the case of TEW, that audience is mostly absent. Physicists on average have little interest in TEW. They are as disinterested in TEW as were geologists in Wegener's proposal. 
For these reasons the Boyd Conjecture should be considered a "trial balloon" rather than an established hypothesis. Until there is a group discussion of interested scholars, this author does not feel assurance that his conjecture is solid. At present it feels wobbly. It is not the Boyd Conjecture that is wobbly, it is Boyd's confidence in the Conjecture that is wobbly.

\section{Implications of the Boyd Conjecture}

Consider the symmetry between QM and TEW. The parity operator $\mathrm{P}$ in QM reflects vectors through the origin. If $\bar{o}$ is a vector then $\mathrm{P}(\overline{\mathrm{o}})=-\overline{\mathrm{o}}$. Thus the operator $\mathrm{P}$ creates a mirror image and simultaneously changes the parity from odd to even or vice versa. Something akin to this kind of symmetry exists in the double slit experiment, when we compare the QM and TEW models. The operator D does the following:

$$
\mathrm{D}(\mathrm{S} \rightarrow \mathrm{X})=\mathrm{X} \rightarrow \mathrm{S} \rightarrow \mathrm{X}
$$

where " $D$ " is the double slit operator, " $S$ " is the particle source, and " $X$ " is the point that appears on the target screen. The left side of the equation is the QM model of how a wave-particle travels from the source to point " $X$ ". The right side of the equation is the TEW model with an elementary wave traveling from " $X$ " to " $S$ " where it triggers (at random) a particle to follow the ray backwards to point "X." The math is identical for both sides of this equation, as we will show in the next two paragraphs. What is different is the mental picture of how things travel through space (see Figure 2 below). The QM model on the left cannot explain what happens in the double slit experiment: no matter how you try to patch up the flaws, there are always unexplained contradictions. We claim that the QM model is a delusion that afflicted science since Thomas Young in 1801, and is a mirror image of how the real world works. Remarkably quantum math developed for the left side of this equation accurately describes the real world, which is shown on the right side of the equation. The parity is the same on both sides of the equation. All that changes when we apply the operator " $D$ " is that delusion changes into realism.

\section{4a. Symmetry of the double slit experiment}

According to the QM model, a wave-particle is fired from the electron gun and arrives at point " $X$ " on the target screen. We define $\mathbf{A}$ as the amplitude of it having come through slit $A$ and the amplitude of having come through slit $B$ is $\mathbf{B}$. The probability of the electron being seen at " $X$ " is $|\mathbf{A}+\mathbf{B}|^{\wedge} \mathbf{2}$. Because these are complex numbers, this probability involves quantum interference.

Next we will apply the operator $D$ to get the TEW explanation. Every point " $X$ " on the target screen radiates elementary rays. We ignore all of them except for those with a deBroglie frequency $\square=E / h$ corresponding to the energy of the electron soon to be emitted. The rays contain and convey zero energy: they are probability amplitudes. The amplitude of an elementary ray starting at point " $X$ " and arriving at the electron gun is $\mathbf{A}+\mathbf{B}$. A multitude of such rays impinge on the gun, one from each point of the target screen, each with a different amplitude. The probability of any specific elementary ray from a specific " $X$ " being seen at the electron gun is $\mid \mathbf{A}$ $+\left.\mathbf{B}\right|^{\wedge} \mathbf{2}$. The electrons choose at random which incident ray to respond to, depending on the amplitude of the incident ray. If an electron is triggered by an incident ray, it will follow that ray backwards with a probability of one. It doesn't matter which slit it is used. There is no interference after the electron is fired. Once fired, the electron will strike the target at "X."

Therefore both models use identically the same math and explain the same pattern on the target screen.

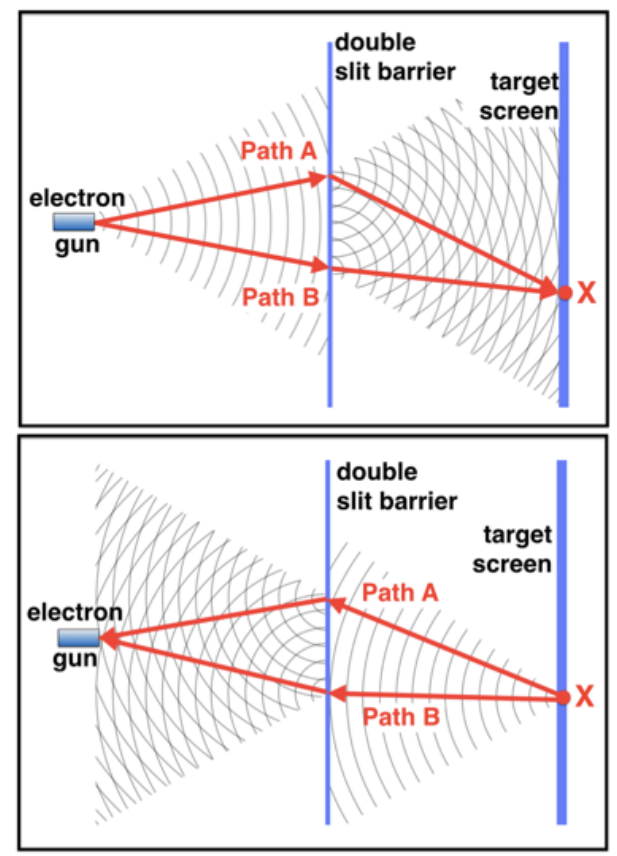


The TEW explanation of the experiment is much simpler than the QM explanation. The TEW model illustrates the Boyd conjecture: that the amplitude of an elementary ray from TEW is the physical analog of a probability amplitude from QM. It also illustrates that wave function collapse occurs much earlier and in a different physical location in TEW compared to QM. In TEW that collapse occurs on the left, at the electron gun, before and during the emission of the electron.

\section{4b. Schrödinger's cat $\&$ the timing of wave function collapse}

The difference of timing of wave function collapse is illustrated by Schrödinger's cat. In both QM and TEW there are two eigenfunctions inside the box: one involves the cat dying from cyanide and the other involves the cat living.

However, wave function collapse occurs at different times. With QM wave function collapse occurs when the lid of the box is opened. The outcome with QM is well known and absurd. With TEW wave function collapse occurs much earlier: at the time of particle emission. There is no interference and no probability amplitude after particle emission. Thus it is the uranium atom that is in a superposition until it decays and emits an alpha particle. The cat is never in a superposition.

When we compare Schrödinger's cat we find that QM and TEW share the same math but a different idea of when wave function collapse occurs. Only the TEW model fits our common sense. The QM model is preposterous: a cat that is both dead and alive simultaneously.

With TEW the "measurement problem" and the "many worlds theory" vanish, because they are irrelevant.

\section{SUMMARY}

The core ideas of TEW are that particles follow zero energy rays backwards. Everywhere in space there are an infinite number of elementary rays of all frequencies, traveling in all directions at the speed of light. Wave particle duality is wrong.

\section{Comparing TEW and QM}

Comparing the two pictures of reality, there are two glaring questions:

1. What is an elementary ray from a QM perspective; and

2. What is a probability amplitude from a TEW perspective?

These two questions are mirror images of one another. Question two answers question one, and vice versa.

This author finds the idea of waves travelling in the opposite direction as particles to be preposterous. But look at the trade-off. Would you rather accept ONE preposterous idea, or live in a completely preposterous universe, such as the one that QM proposes? The problem is that when we present TEW to physicists, they hear the first preposterous idea, stop listening and walk away. Nevertheless, the stone rejected by the builders will become the cornerstone of science before the end of this century. We have on our side a power that cannot be stopped, which is the power of the truth.

How can we compare TEW and QM? They both involve particles and probability amplitudes (known by a different name). One way in which TEW is different from QM is that TEW offers a specific and precise name for that which QM vaguely refers to as "nonlocality." If you want to investigate nonlocal influences, that would be hard to do if they were ill-defined like ghosts, which is the case in QM. In TEW we have a different name for "nonlocality." We call it "elementary waves." These can be crisply defined and it is easy to design experiments to learn more about them. If anyone were interested this author could provide many experimental designs.

A century from now when people look back at our times, they will be puzzled why scientists were so blind to that half of nature that consists of zero energy elementary waves. "What were our ancestors thinking?," our great great grandchildren will ask. Elementary rays will be part of ordinary discourse, the way we all consider Pangea, tectonic plates and convection currents in the earth's magma to be obvious.

Boyd has another conjecture, but it is only half-baked, more of a hunch or intuition than a conjecture. It is that when an elementary ray acquires a particle, it becomes immune to wave interference. Ironically that would mean that the wave interference that we see everywhere in nature is a ripple effect of the particle-less elementary rays that are invisible because they have no energy (because they carry no particles). This would be somewhat like seeing magnetic force lines on a piece of paper that lays on top of a magnet. Only when we sprinkle particles of iron onto the paper does the magnetic flux become visible.

\section{The Future}

What we have discussed so far is only the tip of the iceberg. In the next three paragraphs we will open the door for you to glimpse some of the panorama of unanswered questions about the world of elementary rays. 
If you listen to an introductory lecture on QM, the idea of a probability amplitude will be covered in about five minutes. It took Boyd five years to cover that same territory. There are vast amounts of quantum math that have not been explored from the vantage point of what they tell us about elementary rays.

Then there are the other questions, such as whether there is only one kind of elementary ray, or are there 17 , each corresponding to a different particle in the Standard Model? Where do elementary rays come from? Do they come from empty space or from particles? Is the density of elementary rays in the vacuum of space the same as it is inside a neutron star?

Franco Selleri, the Italian physicist, says that elementary rays move in a medium, which is Lorentz ether at rest. He further says that the rules governing Lorentz ether are different than Einstein's Special Relativity. Selleri's "weak relativity" has become respectable in mainstream physics in Europe. Selleri has published more research about elementary rays than anyone else, although he had them traveling in the wrong direction.(17-28).

\section{REFERENCES}

1. Kuhn, T. S., The Structure of Scientific Revolutions, (Chicago: U. of Chicago Press, 1996).

2. Little, L. E., Theory of Elementary Waves, Physics Essays 9 (1), 100-134 (1996).

3. Little, L. E., "Theory of Elementary Waves," lecture at the Jet Propulsion Labs in 2000, $U R L=h t t p: / /$ tewlip.com/viewtopic. $p h p ? f=8 \& t=18$ (accessdate=May 26, 2015).

4. Little, L. E., Theory of Elementary Waves, (New Classics Library, New York, 2009).

5. J. H. Boyd, "Rethinking a Wheeler delayed choice gedanken experiment," Physics Essays 25, (3) pp. 390-396, 2012. (http://dx.doi.org/10.4006/0836-1398-25.3.390).

6. J. H. Boyd, "Re-thinking a delayed choice quantum eraser experiment: a simple baseball model," Physics Essays, March 2013, Vol. 26, No. 1, pp. 100-109, (doi: 10.4006/0836-1398-26.1.100).

7.

J. H. Boyd, "Re-thinking Alain Aspect's 1982 Bell test experiment with delayed choice," Physics Essays, $26 \quad$ (4) 582-591 (2013). http://physicsessays.org/browse-journal-2/product/39-15-jeffrey-h-boydrethinking-alain-aspect-s-1982-bell-test-experiment-with-delayed-choice.html

8.

J. H. Boyd, "Paul Dirac's view of the Theory of Elementary Waves," Journal of Advances in Physics 13, (2017). in press

9.

J. H. Boyd, "A paradigm shift in mathematical physics, Part 4: Quantum computers and the local realism of all 4 Bell states," Journal of Advances in Mathematics, 11 (4), 5476-5493 (November, 2015). http://cirworld.org/journals/index.php/jam/article/view/5502

10.

J. H. Boyd, "A paradigm shift in mathematical physics, Part 3: A mirror image of Feynman's quantum electrodynamics (QED)," Journal of Advances in Mathematics, 11 (2), 3977-3991 (August, 2015). http://cirworld.com/journals/index.php/jam/article/view/4963

11. J. H. Boyd, "A paradigm shift in mathematical physics, Part 2: A new local realism explains Bell test \& other experiments," Journal of Advances in Mathematics, 10 (9), 3828-3839 (July 2015). http://cirworld.com/journals/index.php/jam/article/view/4806

12.

J. H. Boyd, "A paradigm shift in mathematical physics, Part 1: The Theory of Elementary Waves (TEW)," Journal of Advances in Mathematics $10 \quad$ (9), 3828-3839 (June 2015). http://cirworld.com/journals/index.php/jam/article/view/4719

13. J. H. Boyd, "The Theory of Elementary Waves eliminates Wave Particle Duality," Journal of Advances in Physics 7 (3), 1916-1922 (Feb 2015). http://cirworld.org/journals/index.php/jap/article/view/228n

14.

J. H. Boyd, "A new variety of local realism explains a Bell test experiment: the Theory of Elementary Waves (TEW) with no hidden variables," Journal of Advances in Physics 8 (1), $2051-58$ (Mar 2015) http://cirworld.org/journals/index.php/jap/article/view/252na

15.

J. H. Boyd, "A proposed physical analog of a quantum amplitude: Corkscrew model from the Theory of Elementary Waves (TEW)" Journal of Advances in Physics 10, (3), $2774-2783$ (Oct 2015). http://cirworld.org/journals/index.php/jap/article/view/5116

16. Feynman, Richard P., QED (Oxford University Press and Princeton U. Press, 1985).

17. Selleri, F. Weak Relativity: the physics of space and time without paradoxes. C.Roy Keys Inc, 2009

18. Selleri, F., "Our absolute velocity," in Physical Interpret. of Relativity Theory, (C. Duffy editor), British Soc. Philosophy of Science, London 2002.

19. Selleri, F., "Recovering the Lorentz ether," Apeiron 11, 246 (2004).

20. Selleri, F., "Sagnac effect: end the mystery," in Relativity in Rotating Frames, series of Fundamental Theories of Physics, (A. van der Merwe, editor), Kluwer Academic Publ. (2004). 
21. Selleri, F., "The Sagnac effect, once more," Proceedings of the Natural Philosophy Alliance, volume 11, 525527 (2012).

22. Selleri, F. "Space and time physics with the Lorentz ether: the clock paradox," in, B.G. Sidhnarth, F. Honsell and A. DeAngelis(eds.), Frontiers in Fundamental Physics, pp. 195-208 (Springer, Berlin 2005).

23. Selleri, F., "Noninvariant one-way velocity of light," Foundations of Physics 26, $641-664$ (1996).

24. Selleri, F., "Noninvariant one-way velocity of light and particle collisions," Foundations of Physics Letters $\mathbf{9}$, 43-60 (1996).

25. Freire, Olival, Interview with Dr. Franco Selleri, American Institute of Physics: Oral History Interviews. Accessed 3/21/2017 from: https://www.aip.org/history-programs/niels-bohr-library/oral-histories/28003-1

26. Selleri, F., "On the direct observability of quantum waves," Foundations of Physics 12, no. 11, 1982.

27. Selleri, F., Reply to "Empty waves do not induce stimulated emission in laser media," Physics Letters A 132 , $72-74$ (1988).

28. Selleri, F., Coherence properties of photon amplifiers, Foundations of Physics 17, 739-757(1987).

\section{Author' biography with Photo}

Jeffrey H. Boyd was born into a working class family in 1943 in New Jersey, USA. He is the first member of his family to graduate from college. He has been in dialogue with his cousin, Lewis E. Little, for 60 years. Boyd's undergraduate degree in mathematics was from Brown University in 1965. He has advanced degrees from Harvard, Yale and Case Western Reserve Universities, has served on the faculty of the National Institutes of Health for seven years. His day job is as a psychiatrist, which is fortunate because he need not fear he is risking his family's livelihood by speaking in public about these controversial ideas in physics. Boyd retired after a quarter century at Waterbury Hospital, Waterbury CT, a Yale teaching hospital at which he served as Chairman of Behavioral Health and also Chairman of Ethics. He specialized in treating the chronically mentally ill, most of whom were indigent or homeless. He published research in the areas of epidemiology, psychiatric diagnoses, firearms suicide, and wrote a book about how to live with debilitating illness: Being Sick Well. He has published research in the New England Journal of Medicine, Journal of Advances in Mathematics and Physics Essays, and served as a reviewer for the Canadian Journal of Physics. He has given scholarly lectures on TEW at the American Physical Society more than a dozen times. Boyd thinks the future of physics belongs to those who are now teenage geeks. That generation watches videos rather than reading. The study in Boyd's house is arranged like a television studio with a green screen, and he spends his free time creating amusing five minute YouTube videos to teach TEW. Currently he is downloading music videos that have been watched more than a billion times, and rearranging the content so as to teach ideas from TEW in a format that might capture the imagination of teenage nerds. Boyd finds that his geriatric friends love these teenage videos! Slowly his peculiar ideas are gaining popularity: More than 42,000 people have watched his YouTube videos.

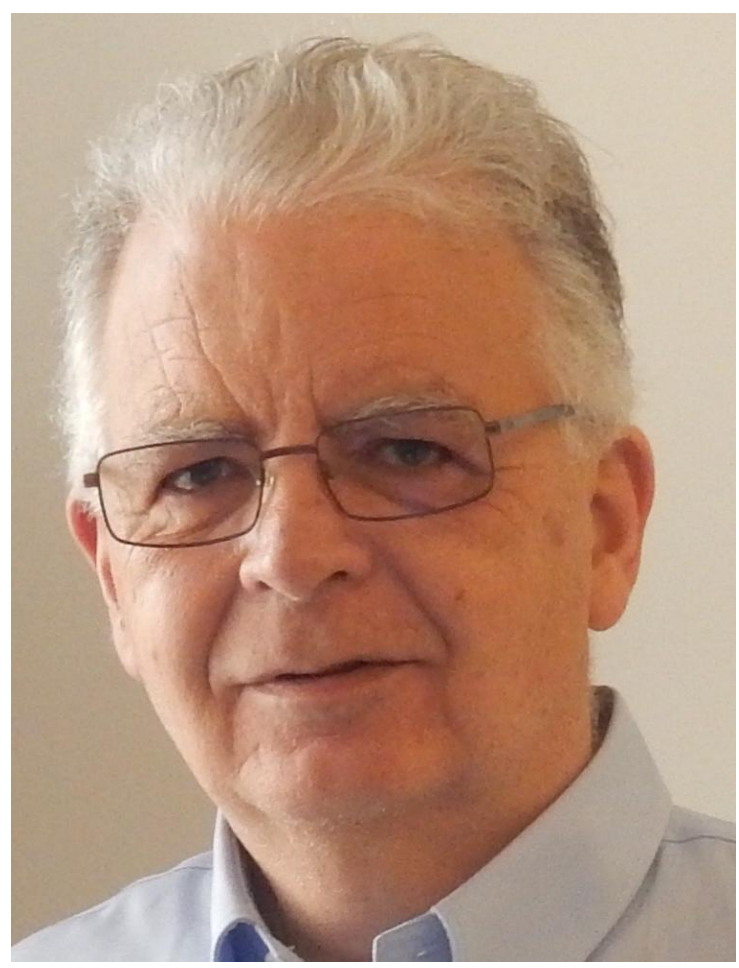

